\title{
The Thioredoxin Reductase-1 Inhibitor Aurothioglucose Attenuates Lung Injury and Improves Survival in a Murine Model of Acute Respiratory Distress Syndrome
}

\author{
Rodney D. Britt, Jr.,"* Markus Velten,,"* Morgan L. Locy,, Lynette K. Rogers, ${ }^{1,3}$ and Trent E. Tipple ${ }^{1,3}$
}

\begin{abstract}
Aims: Inflammation and oxygen toxicity increase free radical production and contribute to the development of acute respiratory distress syndrome (ARDS), which is a significant cause of morbidity and mortality in intensive care patients. We have previously reported increased glutathione (GSH) levels in lung epithelial cells in vitro and attenuated adult murine hyperoxic lung injury in vivo after pharmacological thioredoxin reductase-1 (TrxR1) inhibition. Using a murine ARDS model, we tested the hypothesis that aurothioglucose (ATG) treatment increases pulmonary GSH levels, attenuates lung injury, and decreases mortality in a GSHdependent manner. Results: Adult mice received a single intratracheal dose of $0.375 \mu \mathrm{g} / \mathrm{g}$ lipopolysaccharide (LPS) $12 \mathrm{~h}$ before a single intraperitoneal injection of $25 \mathrm{mg} / \mathrm{kg}$ ATG. Control mice received intratracheal and/or intraperitoneal saline. Mice were then exposed to room air or hyperoxia $\left(>95 \% \mathrm{O}_{2}\right)$. Lung injury was assessed by bronchoalveolar lavage protein concentrations. Expression of glutamate-cysteine ligase modifier subunit (GCLM), GSH, cytokines, and chemokines was determined. Exposure to LPS/hyperoxia induced inflammation and lung injury. ATG treatment significantly attenuated lung injury, increased lung GCLM expression and GSH levels, and decreased mortality. GSH depletion completely prevented the protective effects of ATG in LPS/hyperoxia-exposed mice. Innovation: ATG treatment significantly attenuates lung injury and enhances survival in a clinically relevant murine model of ARDS. The protective effects of ATG are GSH dependent. Conclusion: Augmentation of GSH systems by TrxR1 inhibition could represent a promising therapeutic approach to attenuate oxidant-mediated lung injury and improve patient outcomes. Antioxid. Redox Signal. 20, 2681-2691.
\end{abstract}

\section{Introduction}

A CUTE RESPIRATORY DISTRESS SYNDROME (ARDS) is a significant cause of morbidity and mortality among critically ill patients (37). Many patients require intensive care as a consequence of lung injury brought about by infectious and/or inflammatory etiologies. These patients often require treatment with supraphysiologic $\mathrm{O}_{2}$ levels to maintain adequate tissue oxygenation. Unfortunately, inflammation and hyperoxia increase free radical production and contribute to the development of ARDS (43).
Thioredoxin reductase-1 (TrxR1) is an NADPH-dependent oxidoreductase that is best known for reducing the active site of oxidized thioredoxin-1 (Trx1) (2). Aurothioglucose (ATG) and auranofin $(\mathrm{AFN})$, anti-inflammatory gold compounds that are approved to treat rheumatoid arthritis, are potent TrxR1 inhibitors (18). We have previously shown that ATG treatment of adult mice is well tolerated, preserves lung glutathione (GSH) levels, and attenuates hyperoxic lung injury (42). The protective effects of ATG against hyperoxic lung injury were absent in mice with a genetically impaired GSH system (42). We recently demonstrated that the protective effects of TrxR1

\footnotetext{
${ }^{1}$ Center for Perinatal Research, The Research Institute at Nationwide Children's Hospital, Columbus, Ohio.

${ }^{2}$ Department of Anesthesiology and Intensive Care Medicine, Rheinische Friedrich-Wilhelms-University, University Medical Center, Bonn, Germany.

${ }^{3}$ Department of Pediatrics, College of Medicine, The Ohio State University, Columbus, Ohio.

*These authors contributed equally to this study.
} 


\section{Innovation}

We previously found that thioredoxin reductase-1 (TrxR1) inhibition activated nuclear factor E2-related factor 2 (Nrf2)-dependent antioxidant responses and increased glutathione (GSH) levels in vitro and in vivo. The present studies demonstrate that mice treated with the TrxR1 inhibitor aurothioglucose (ATG) had significantly enhanced lung GSH levels, attenuated lung injury, and enhanced survival in a murine acute respiratory distress syndrome model combining intratracheal lipopolysaccharide with prolonged hyperoxic exposure. Furthermore, the protective effects of ATG were GSH dependent. If pharmacologic TrxR1 inhibition can be safely utilized to induce Nrf2 activation and increase GSH levels, this novel strategy could attenuate lung injury and improve outcomes in critically ill patients.

inhibitors are likely mediated via nuclear factor E2-related factor 2 (Nrf2) activation with subsequent increases in GSH levels (23). GSH depletion markedly enhanced hyperoxic susceptibility in AFN-treated murine lung epithelial cells in vitro (23). Thus, our data support our current working model in which the protective effects of TrxR1 inhibitors are GSH dependent.

In acute lung injury (ALI) models, bacterial or acid-induced lung injury is further exacerbated by hyperoxic exposure $(1,6$, $21,22,27)$. Thus, the present studies utilized an adult murine ARDS model which combines inflammatory and hyperoxic exposures to test the hypothesis that ATG treatment attenuates lung inflammation and injury, increases pulmonary GSH levels, and improves survival. Mice received a single intratracheal dose of Escherichia coli lipopolysaccharide (LPS) in saline $12 \mathrm{~h}$ before the administration of a single intraperitoneal injection of $25 \mathrm{mg} / \mathrm{kg}$ ATG. Control mice received intratracheal and/or intraperitoneal saline. Mice were then exposed to room air (RA) or hyperoxia $\left(>95 \% \mathrm{O}_{2}\right)$. Lung injury was more severe in LPS/hyperoxia-exposed mice compared with saline/ RA or saline/hyperoxia-exposed mice. In LPS/hyperoxiaexposed mice, ATG treatment significantly attenuated lung injury and increased lung GSH levels. Most importantly, ATG treatment significantly improved survival in LPS/hyperoxiaexposed mice. GSH depletion with buthionine sulfoximine (BSO) completely prevented the protective effects of ATG treatment in mice exposed to both LPS and hyperoxia.

\section{Results}

LPS and hyperoxic exposure increase protein concentrations, cell numbers, and pro-inflammatory cytokine/chemokine expression in bronchoalveolar lavage fluids

Mice were treated with intratracheal saline or LPS $12 \mathrm{~h}$ before exposure to RA or hyperoxia $\left(>95 \% \mathrm{O}_{2}\right)$ for $24 \mathrm{~h}$. Thus, there were four treatment groups: saline/RA, saline/ hyperoxia, LPS/RA, and LPS/hyperoxia (Fig. 1). Independent effects of LPS and hyperoxia on bronchoalveolar lavage (BAL) cell number were detected (Fig. 2A). BAL cell numbers in LPS/hyperoxia-exposed mice were greater than in all other groups. An independent effect of LPS on BAL protein concentrations was also detected. BAL protein con-

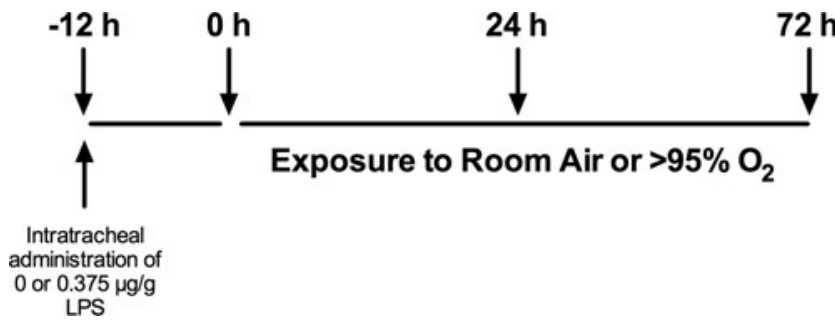

FIG. 1. Experimental design. Protocol for experiments in Figures 2-4.

centrations were greater in LPS/hyperoxia-exposed mice than in saline/RA controls and saline/hyperoxia-exposed mice, respectively. We next determined the impact of LPS and/or hyperoxic exposure on pro-inflammatory cytokine and chemokine expression. BAL fluids were collected after exposure to hyperoxia for $24 \mathrm{~h}$. Concentrations of keratinocyte-derived chemokine (KC), macrophage inflammatory protein-2 (MIP2 ), interleukin-6 (IL-6), tumor necrosis factor- $\alpha$ (TNF $\alpha$ ), and receptor for advanced glycosylation end products (RAGE) in BAL fluids were determined. An independent effect of LPS was detected on all analytes, while an independent effect of hyperoxia was detected for only IL-6 and RAGE (Fig. 2B). $\mathrm{KC}, \mathrm{MIP}-2$, IL-6, and TNF $\alpha$ concentrations were greater in the LPS/RA and LPS/hyperoxia groups than in saline/RA and saline/hyperoxia groups at $24 \mathrm{~h}$. IL-6 levels were highest in the LPS/hyperoxia group compared with all other treatment groups, while $\mathrm{KC}$ expression was greater in all three other treatment groups than in saline/RA controls.

To characterize the effects of prolonged hyperoxic exposure in our model, mice were treated with intratracheal saline or LPS $12 \mathrm{~h}$ before exposure to RA or hyperoxia $\left(>95 \% \mathrm{O}_{2}\right)$ for $72 \mathrm{~h}$. An independent effect of LPS on BAL cell number was detected (Fig. 3A). BAL cell numbers in both LPS/RA and $\mathrm{LPS} / \mathrm{O}_{2}$ treatment groups were greater than in saline/RA controls. An independent effect of LPS on BAL protein concentrations was detected, though there were no individual differences. We next determined the impact of LPS and/or hyperoxic exposure on pro-inflammatory cytokine and chemokine expression. Concentrations of KC, MIP-2, IL-6, $\mathrm{TNF} \alpha$, and RAGE in BAL fluids were determined after exposure to hyperoxia for $72 \mathrm{~h}$ (Fig. 3B). Independent effects of LPS and hyperoxia on KC and IL-6 were detected, whereas an effect of LPS was detected on RAGE levels. IL-6 and RAGE levels were highest in the LPS/hyperoxia group compared with all other treatment groups, while $\mathrm{KC}$ expression was greater in all three other treatment groups than in saline/RA controls.

\section{LPS and hyperoxic exposure alter GSH levels in lung tissues}

To assess the effect of LPS and/or hyperoxia on the lung GSH system, GSH and GSH disulfide (GSSG) levels in tissue homogenates were determined at 24 or $72 \mathrm{~h}$. GSH/GSSG ratios were then calculated using these values. An effect of hyperoxia on GSH levels was detected at both 24 and $72 \mathrm{~h}$ (Fig. 4A). Hyperoxia and LPS/hyperoxia dramatically increased GSH levels, whereas lung GSH levels were $60 \%$ lower in lungs from LPS-treated mice than in saline-treated controls at $24 \mathrm{~h}$. Lung $\mathrm{GSH} / \mathrm{GSSG}$ ratios were not different at $24 \mathrm{~h}$. At $72 \mathrm{~h}$, an effect 


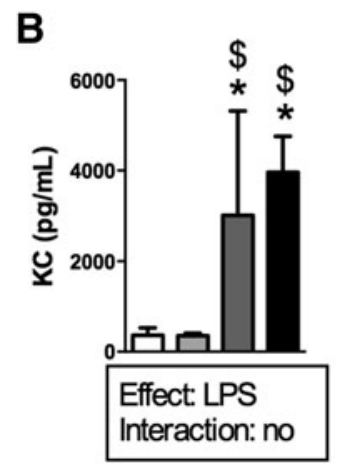

A

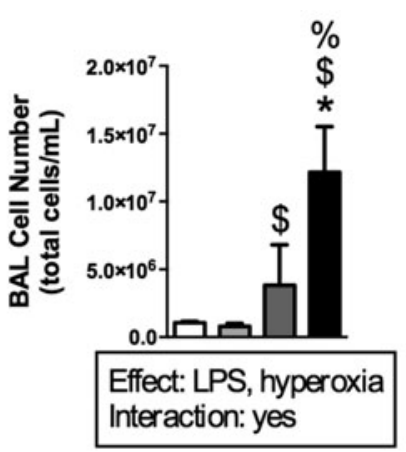

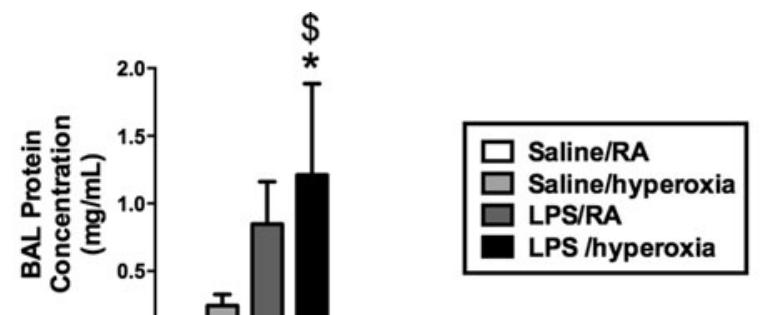

Effect: LPS Interaction: no
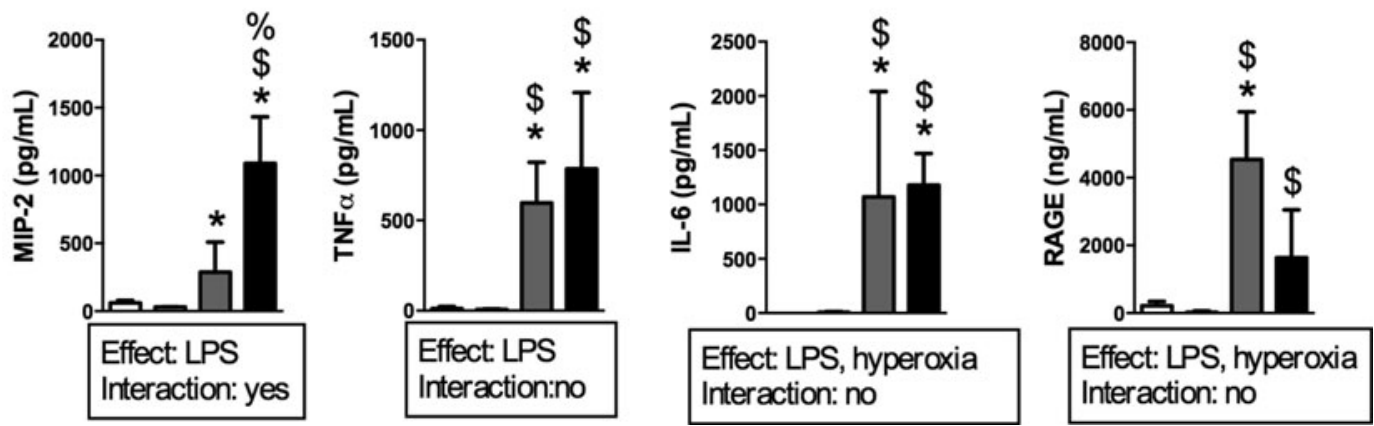

FIG. 2. BAL protein concentrations, cell counts, cytokines, and chemokine levels after exposure to LPS and hyperoxia for $\mathbf{2 4} \mathbf{~ h}$. Adult mice received intratracheal saline or LPS at $12 \mathrm{~h}$ before exposure to $\mathrm{RA}$ or hyperoxia $\left(>95 \% \mathrm{O}_{2}\right)$ for $24 \mathrm{~h}$ as described in the Materials and Methods section. (A) Cell numbers were determined using trypan blue exclusion, and protein concentrations were measured by Bradford assay. Data (mean \pm SD) were analyzed by two-way ANOVA with Tukey's multiple-comparisons test post hoc $(n=3-6)$. ${ }^{*} p<0.01$ versus saline/RA, ${ }^{\$} p<0.01$ versus saline/hyperoxia, and ${ }^{\%} p<0.05$ versus LPS/RA. (B) Concentrations of KC, MIP-2, IL-6, TNF $\alpha$, and RAGE were measured by ELISA. Data (mean $\pm \mathrm{SD})$ were analyzed by two-way ANOVA with Tukey's multiple-comparisons test post hoc $(n=3-6)$. ${ }^{*} p<0.03$ versus saline/RA, ${ }^{\$} p<0.02$ versus saline/hyperoxia, and ${ }^{\%} p<0.01$ versus LPS/RA. RA, room air; BAL, bronchoalveolar lavage; IL-6, interleukin-6; KC, keratinocyte-derived chemokine; LPS, lipopolysaccharide; MIP-2, macrophage inflammatory protein-2; RAGE, receptor for advanced glycosylation end products; TNF $\alpha$, tumor necrosis factor- $\alpha$.

of hyperoxia on GSSG levels was detected (Fig. 4B). In saline/ hyperoxia and in LPS/hyperoxia-exposed mice, lung GSSG levels were significantly greater than in saline/RA-exposed mice. Furthermore, an effect of hyperoxia on lung GSH/GSSG ratios was also detected at $72 \mathrm{~h}$. Lung GSH/GSSG ratios were fivefold lower in saline/hyperoxia mice than in saline/RA mice. In LPS/hyperoxia mice, lung GSH/GSSG ratios were fourfold lower than in LPS/RA mice.

\section{ATG treatment decreases BAL protein concentrations but does not alter pro-inflammatory cytokine/chemokine expression in LPS/hyperoxia-exposed mice}

To test the hypothesis that ATG treatment would attenuate lung injury in our inflammation/hyperoxia ARDS model, adult mice were injected with saline or $25 \mathrm{mg} / \mathrm{kg}$ ATG at $12 \mathrm{~h}$ after intratracheal LPS administration (Fig. 5). Mice were then exposed to hyperoxia for $72 \mathrm{~h}$. Though we detected no effect of ATG on BAL cell numbers, BAL protein concentrations were significantly lower in ATG-treated mice than in saline-treated controls (Fig. 6A). We next examined the impact of ATG treatment on the levels of KC, MIP-2, IL-6, $\mathrm{TNF} \alpha$, and RAGE in BAL fluids. There were no differences in the expression of pro-inflammatory cytokine and chemokine expression between ATG-treated and saline-treated groups (Fig. 6B).

\section{ATG treatment enhances GCLM transcription}

and increases GSH levels in BAL cells

and lung tissues of LPS/hyperoxia-exposed mice

The impact of ATG on the GSH system in LPS/hyperoxiaexposed mice was determined by measuring glutamate-cysteine ligase modifier subunit (GCLM) mRNA expression and GSH levels. Compared with saline-treated controls, ATG treatment significantly enhanced GCLM expression (Fig. 7A). GSH levels in BAL cells from ATG-treated animals were approximately thrice higher than in cells from saline-treated controls (Fig. 7B). ATG treatment also significantly enhanced GSH levels in lung tissues (Fig. 7C) but had no effect on GSSG levels. Lung tissue GSH/GSSG ratios were calculated; however, no effect of ATG was detected by the statistical methods used $(p=0.058)$.

\section{ATG treatment enhances survival}

in LPS/hyperoxia-exposed mice

and the protective effects are prevented by BSO

Given the positive effects on ATG on BAL protein concentrations, BAL cell GSH, and lung GSH levels, we hypothesized that ATG treatment would improve the survival of LPS/hyperoxia-exposed mice. Furthermore, we suspected that any protective effects would be GSH dependent. To test this hypothesis, adult mice were treated with $25 \mathrm{mg} / \mathrm{kg}$ ATG and/or $800 \mathrm{mg} / \mathrm{kg}$ BSO $12 \mathrm{~h}$ after intratracheal LPS 


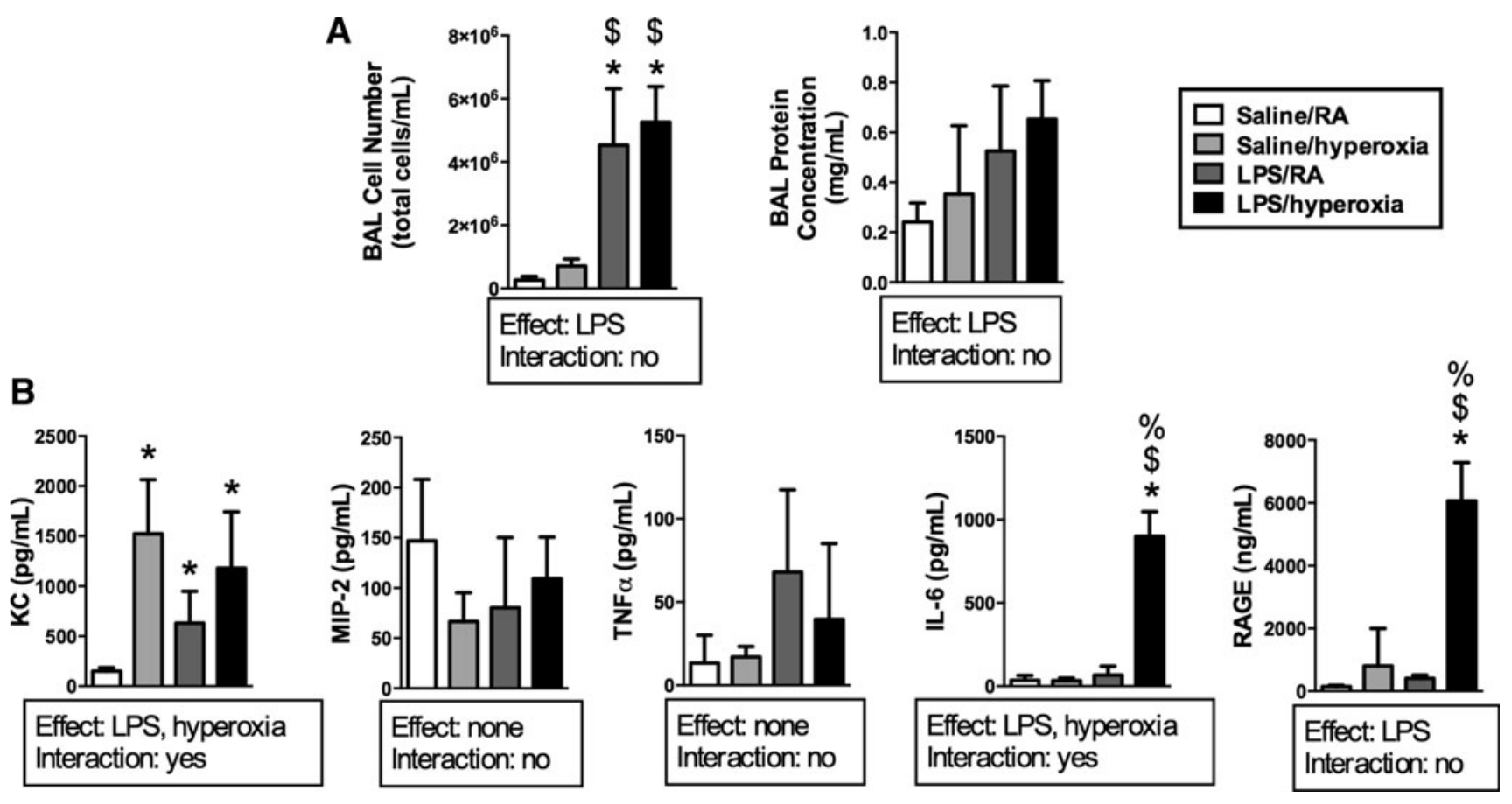

FIG. 3. BAL protein concentrations, cell counts, cytokines, and chemokine levels after exposure to LPS and hyperoxia for $72 \mathrm{~h}$. Adult mice received intratracheal saline or LPS at $12 \mathrm{~h}$ before exposure to RA or hyperoxia $\left(>95 \% \mathrm{O}_{2}\right)$ for $72 \mathrm{~h}$ as described in the Materials and Methods section. (A) Cell numbers were determined using trypan blue exclusion, and protein concentrations were measured by Bradford assay. Data (mean \pm SD) were analyzed by two-way ANOVA with Tukey's multiple-comparisons test post hoc $(n=3-6) . * p<0.0001$ versus saline/RA, ${ }^{\$} p<0.02$ versus saline/hyperoxia. (B) Concentrations of KC, MIP- 2 , IL-6, TNF $\alpha$, and RAGE were measured by ELISA. Data (mean \pm SD) were analyzed by two-way ANOVA with Tukey's multiple-comparisons test post hoc $(n=3-6) . * p<0.005$ versus saline/RA, ${ }^{\$} p<0.0002$ versus saline/hyperoxia, and ${ }^{\%} p<0.0002$ versus LPS/RA.

administration and were subsequently exposed to hyperoxia. Mice were euthanized on displaying prospectively defined end point criteria (see Materials and Methods section), because our institution does not allow "death as an endpoint" studies. Thus, there were four treatment groups: saline, BSO, ATG, and ATG/ BSO (Fig. 8A). Median survival of saline-treated controls $(105 \mathrm{~h})$ was not different than mice treated with BSO alone (101 h). ATG treatment significantly enhanced survival (median survival $150 \mathrm{~h}$ ) with two healthy appearing ATG-treated mice still alive at the termination of these studies (Fig. 8B). BSO treatment completely prevented the protective effects of ATG in LPS/hyperoxia-exposed mice (median survival of $29 \mathrm{~h}$ ) with no mouse surviving beyond $35 \mathrm{~h}$. To determine the impact of ATG on lung injury, we performed hematoxylin and eosin (H\&E) staining on paraffin-embedded lungs at $72 \mathrm{~h}$. Representative images are shown in Figure 9A. Standardized histology scoring in saline, BSO, and ATG treated-mice was performed as described in the Materials and Methods section to formally quantify lung injury (Fig. 9B). The most profound effects of LPS $/ \mathrm{O}_{2}$, including the presence of hyaline membranes and bronchiolar epithelial necrosis, were identified in lung sections from saline-treated mice. These findings were absent in the ATG- and BSO-treated mice. Lung histology scores were highest in saline-treated mice. Both ATG and BSO treatment were associated with significantly lower scores at $72 \mathrm{~h}$.

\section{Discussion}

Many patients who develop ARDS require hyperoxic therapy as a consequence of lung injury brought about by infectious and/or inflammatory etiologies. To more closely mimic clinical ARDS, we tested the efficacy of the TrxR1 inhibitor ATG in a lung injury model that combined inflammation (LPS) and hyperoxia. The major findings in the present studies in adult mice are as follows: (i) Lung injury after LPS/hyperoxia exposure is characterized by pulmonary edema, inflammation, and decreased lung GSH/GSSG ratios; (ii) ATG treatment after LPS administration and before hyperoxic exposure decreased pulmonary edema and enhanced lung GSH systems but had no effect on pro-inflammatory cytokine/chemokine expression; (iii) ATG treatment significantly attenuated lung injury and improved survival; and (iv) the protective effects of ATG were completely prevented by BSO. Thus, our novel studies are the first to demonstrate that ATG-mediated TrxR1 inhibition attenuates lung injury and enhances survival in a severe ARDS model and that these effects appear to be GSH dependent.

Our findings of increased lung edema, inflammation, and pro-inflammatory cytokine/chemokine levels in LPS/RA mice are consistent with those published by Aggarwal et al. (1). RAGE levels are increased in ALI patients and are an indirect indicator of lung injury in experimental models (40). The highest RAGE levels and, by extrapolation, the highest degree of lung injury at $24 \mathrm{~h}$ of exposure, was found in LPS/RA mice. Inflammation causes enhanced reactive oxygen species (ROS) production and decreased GSH levels, resulting in upregulation of both pro-inflammatory and antioxidant genes (35). Transcription factors that regulate proinflammatory cytokines and chemokines are sensitive to GSH levels, and GSH depletion enhances pro-inflammatory 
A
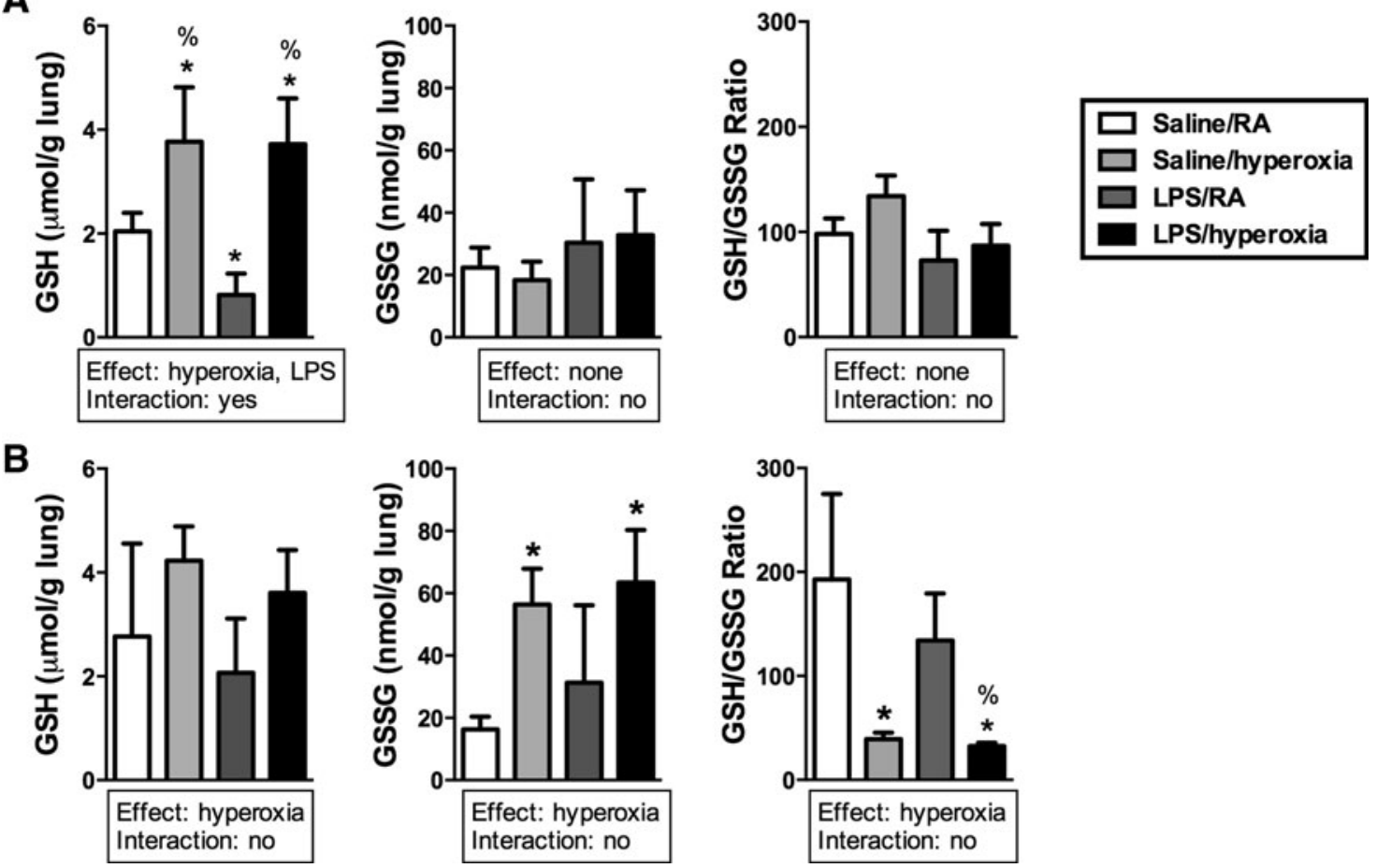

FIG. 4. GSH and GSSG levels in lung tissues after exposure to LPS and hyperoxia for $\mathbf{2 4}$ or $\mathbf{7 2} \mathrm{h}$. Adult mice received intratracheal saline or LPS at $12 \mathrm{~h}$ before exposure to RA or hyperoxia $\left(>95 \% \mathrm{O}_{2}\right)$. (A) At $24 \mathrm{~h}, \mathrm{GSH}$ and GSSG levels were determined and GSH/GSSG ratios were calculated as described in the Materials and Methods section. Data (mean $\pm \mathrm{SD})$ were analyzed by two-way ANOVA with Tukey's multiple-comparisons test post hoc $(n=3-5)$. * $p<0.002$ versus saline/RA, ${ }^{\%} p<0.0006$ versus $\mathrm{LPS} / \mathrm{RA}$. (B) At $48 \mathrm{~h}$, GSH and GSSG levels were determined and GSH/GSSG ratios were calculated as described in the Materials and Methods section. Data (mean $\pm \mathrm{SD}$ ) were analyzed by two-way ANOVA with Tukey's multiple-comparisons test post hoc $(n=3-5) .{ }^{*} p<0.04$ versus saline/RA, ${ }^{\%} p<0.02$ versus LPS/RA. GSH, glutathione; GSSG, GSH disulfide.

signaling in the alveolar epithelium $(19,36)$. Our data in LPS/ RA mice indicated that lung GSH levels were significantly decreased at $24 \mathrm{~h}$ of exposure ( $36 \mathrm{~h}$ after LPS administration) but recovered by $72 \mathrm{~h}$ ( $84 \mathrm{~h}$ after LPS administration). Furthermore, the restoration of GSH levels was associated with normalization of cytokine, chemokine, and RAGE levels at $72 \mathrm{~h}$. Thus, we speculate that in LPS/RA mice, ROS-mediated induction of GSH synthesis, likely via Nrf2 activation, restored GSH levels which attenuated pro-inflammatory signaling, enabling the resolution of lung injury.

Lung edema, inflammation, and pro-inflammatory cytokine levels were also elevated in LPS/hyperoxia mice at $24 \mathrm{~h}$. Only BAL cell numbers, KC and IL-6 levels remained elevated at $72 \mathrm{~h}$ in LPS/hyperoxia-exposed mice. These findings suggest a synergistic effect of LPS and hyperoxia similar to

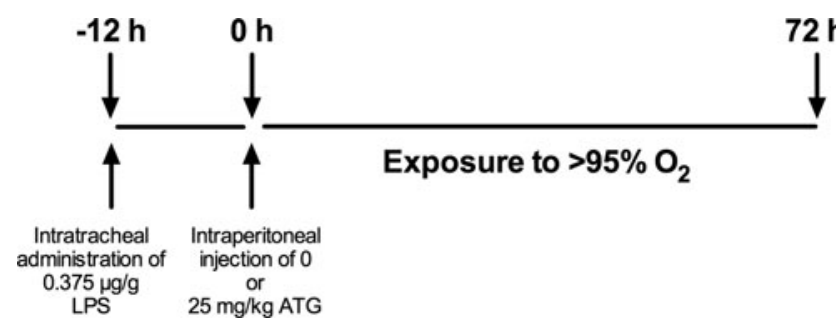

FIG. 5. Experimental design. Protocol for experiments in Figures 6-7. that reported in isolated peritoneal macrophages and in a rat model of bronchopulmonary dysplasia $(9,20)$. Alternatively, since we measured cytokine and chemokine concentrations in BAL fluids, differences could be also attributed to alterations in cellular secretion. The highest GSH levels at $24 \mathrm{~h}$ were seen in saline/hyperoxia and LPS/hyperoxia mice. Thus, hyperoxia appeared to be a much stronger stimulus for GSH production than did LPS alone. Continuous hyperoxic exposure likely results in continuous ROS generation, GSH consumption, and persistent activation of GSH synthetic pathways. Thus, we speculate that hyperoxia induces sustained ROS production, leading to enhanced Nrf2 activation and maximal GSH synthesis. We interpret the lack of differences in lung GSH levels in both hyperoxia-exposed groups at 24 and $48 \mathrm{~h}$ to indicate maximal activation of GSH synthesis. Continued increases in RAGE levels in LPS/hyperoxia mice between 24 and $72 \mathrm{~h}$ suggest that GSH production is insufficient to arrest the progression of lung injury in the setting of persistent oxidative stress.

We previously reported that ATG pre-treatment attenuated hyperoxic lung injury in $\mathrm{C} 3 \mathrm{H} / \mathrm{HeN}$ mice; however, this effect was unanticipated and was not the objective of those studies (42). Our previous studies also revealed that GSH levels and GSH/GSSG ratios were preserved in ATG-treated mice, though the mechanism was not clear at that time. We later determined a plausible mechanism, Nrf2 activation, for the protective effect of ATG, and our studies in vitro indicated 

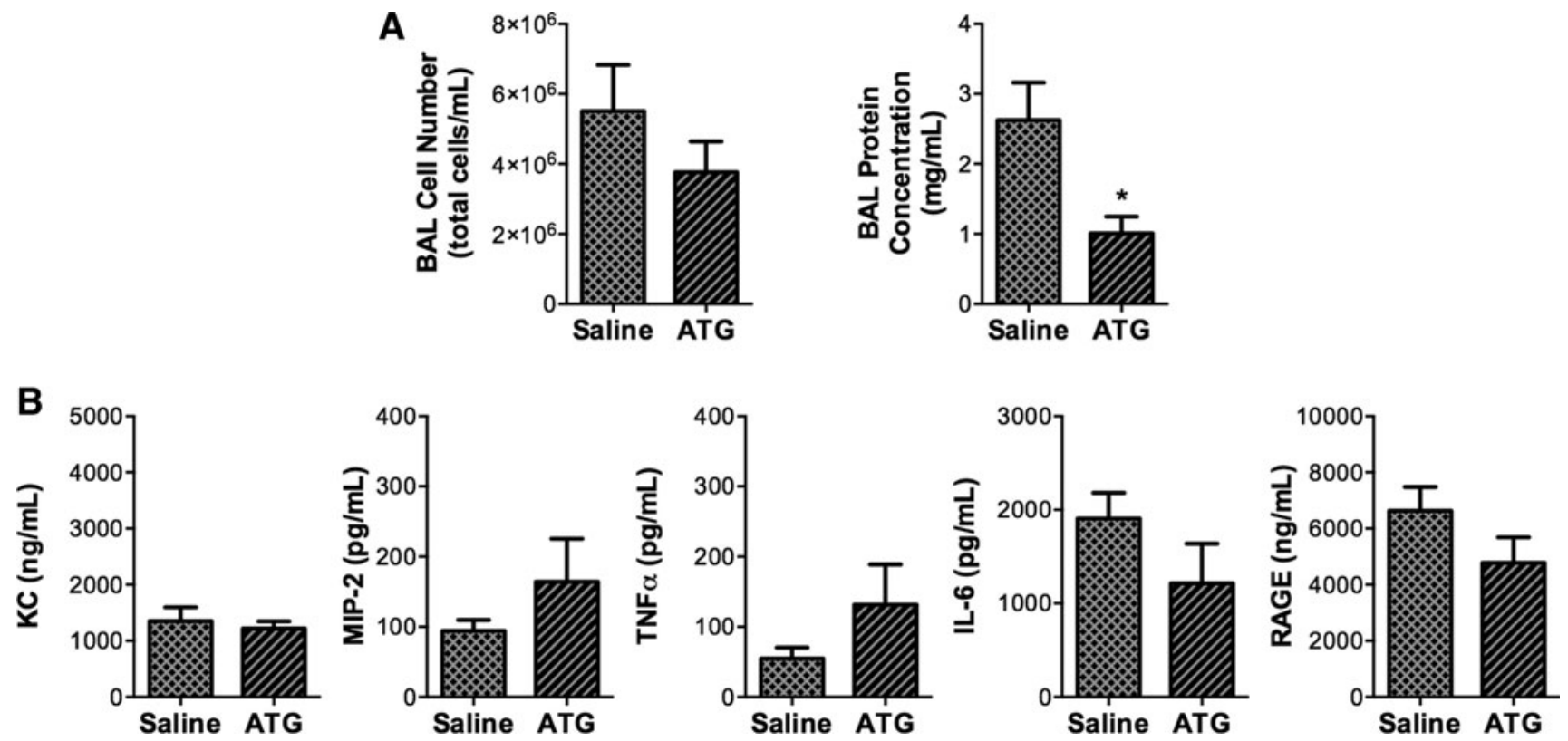

FIG. 6. Effects of ATG treatment on protein concentrations, cell numbers, and cytokine or chemokine expression in BAL fluids from LPS/hyperoxia-exposed mice. Adult mice received a single intraperitoneal injection of saline or $25 \mathrm{mg} /$ $\mathrm{kg}$ ATG at $12 \mathrm{~h}$ after intratracheal LPS administration and were then exposed to hyperoxia $\left(>95 \% \mathrm{O}_{2}\right)$ for $72 \mathrm{~h}$ as described in the Materials and Methods section. (A) Cell numbers were determined using trypan blue exclusion, and protein concentrations were measured by Bradford assay. (B) Levels of KC, MIP-2, IL-6, TNF $\alpha$, and RAGE were measured in BAL by ELISA. Data (mean $\pm \mathrm{SD})$ were analyzed by unpaired Student's $t$-test $(n=6-8)$. ${ }^{*} p=0.011$ versus saline. ATG, aurothioglucose.
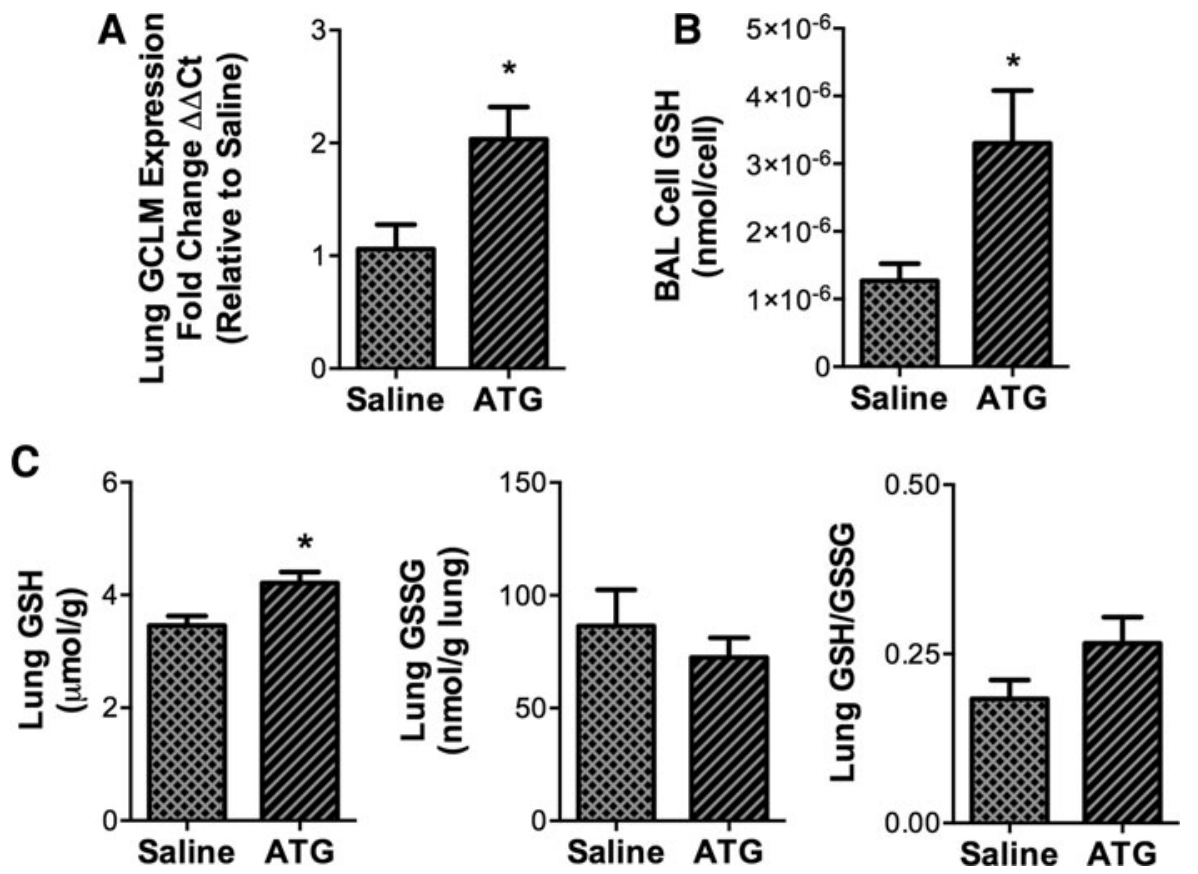

FIG. 7. Effects of ATG treatment on GCLM expression, GSH, and GSSH levels in lung BAL and tissues from LPS/ hyperoxia-exposed mice. Adult mice received a single intraperitoneal injection of saline or $25 \mathrm{mg} / \mathrm{kg}$ ATG at $12 \mathrm{~h}$ after intratracheal LPS administration and were then exposed to hyperoxia $\left(>95 \% \mathrm{O}_{2}\right)$ for $72 \mathrm{~h}$ as described in the Materials and Methods section. (A) GCLM mRNA levels in lung tissues were determined by qRT-PCR and were normalized to $\beta$-actin. GSH levels in (B) BAL cell lysates and GSH and GSSG levels in (C) lung tissues were determined as described in the Materials and Methods section. Data (mean $\pm \mathrm{SD})$ were analyzed by unpaired Student's $t$-test $(n=3-7)$. * $p<0.05$ versus saline. Lung GSH/GSSG ratios were calculated but were not different from each other $(p=0.058)$. GCLM, glutamatecysteine ligase modifier subunit; qRT-PCR, quantitative reverse transcriptase polymerase chain reaction. 

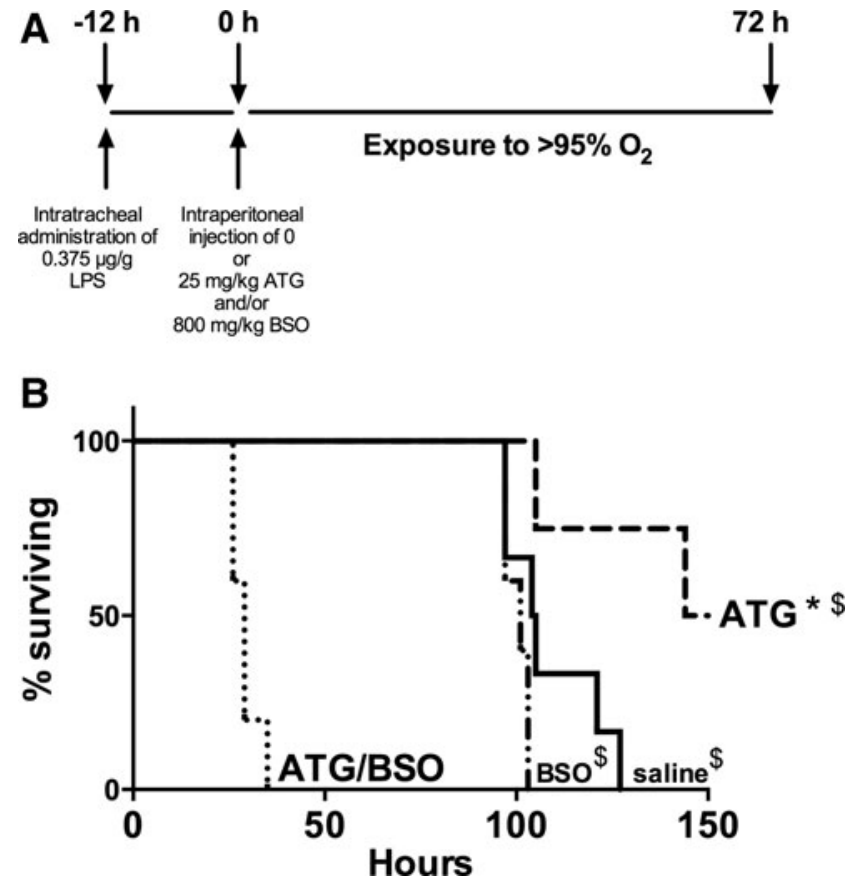

FIG. 8. Effects of ATG and BSO on survival of LPS/ hyperoxia-exposed mice. (A) Protocol for experiments in Figures $8 \mathrm{~B}$ and 9. Adult mice were treated with a single intraperitoneal injection of saline, $25 \mathrm{mg} / \mathrm{kg}$ ATG, and/or $800 \mathrm{mg} / \mathrm{kg}$ BSO at $12 \mathrm{~h}$ after intratracheal LPS administration. Mice were then exposed to hyperoxia and were euthanized on displaying end point criteria as described in the Materials and Methods section. Thus, there were four treatment groups: saline, BSO, ATG, and ATG/BSO. (B) Data were analyzed by Log-rank (Mantel-Cox) test $(n=4-6)$. ${ }^{*} p=0.023$ versus saline; ${ }^{\$} p<0.005$ versus ATG/BSO. BSO, buthionine sulfoximine.

that the effect was GSH dependent (23). In order to determine the efficacy of ATG as an intervention in a more clinically relevant murine lung injury model, we administered ATG after the initiation of a mild inflammatory response but before hyperoxic exposure. Though ATG had no effect on BAL cell counts or chemokine/cytokine levels, it significantly attenuated pulmonary edema. The effects of ATG on chemokine/ cytokine levels may be under-represented due to greater pulmonary edema in saline-treated LPS/hyperoxia mice. Since chemokine/cytokines are determined per unit volume, a dilution effect is likely. Nonetheless, higher lung GCLM expression, tissue GSH levels, and BAL cell GSH levels in ATG-treated mice suggest that ATG stimulated GSH synthesis. Most importantly, ATG significantly decreased lung injury and enhanced the survival of LPS/hyperoxia mice.

Hyaline membranes and bronchiolar epithelial necrosis were present in lung sections from saline-treated mice exposed to hyperoxia for $72 \mathrm{~h}$, whereas the absence of these findings in ATG-treated lungs was similar to our previous findings in mice exposed to hyperoxia alone (42). Right lung/ body weight ratios between these three groups did not differ at $72 \mathrm{~h}$, which is consistent with our findings in $\mathrm{C} 3 \mathrm{H} / \mathrm{HeN}$ mice exposed to hyperoxia alone. Rodent models of adult hyperoxic lung injury have been extensively characterized over the past 20 years (13-16). In adult mice, the course of lung injury passes a "point of no return," where intervention or therapies are not able to reverse the damage and death is imminent. To investigate the mechanisms associated with our intervention, which change the course of injury, we elected to study animals at $72 \mathrm{~h}$, a time point proximal to that at which hyperoxic injury is irreversible. Lung weight to body weight ratios are not expected to be different at this time point, because overt tissue death has not yet occurred $(7,8,17)$. Had we chosen a later time point, it is likely that differences in right lung/body weight ratios would be detectable later in course of hyperoxic exposure as was found in our previous studies (42). We interpret our data to indicate that ATG altered the time course of fatal lung injury as demonstrated by increased survival. We speculate that ATG induces endogenous GSH-dependent responses that attenuate tissue injury and lung damage. Simultaneous administration of BSO blocked the protective effect of ATG in LPS/hyperoxia mice. This finding is similar to our previous studies in vitro and confirms the importance of GSH toward ATG-mediated protection. Treatment with BSO alone had no significant effect on mortality in our studies, and histologic evidence of lung injury was less than in saline-treated mice. These findings are likely due to compensation by the Trx system as previously described (42).

Our previous studies implicated sustained Nrf2 activation as the mechanism by which ATG-dependent TrxR1 inhibition confers protection against hyperoxic lung injury. Further, our previous studies also demonstrated that the protective effects of ATG are GSH dependent (23). In the current studies, the poorest survival in LPS/hyperoxia-exposed mice was observed in mice treated with the combination of ATG and BSO. Indeed, these data are consistent with our earlier findings of profound mortality in ATG-treated GSH reductase knockout mice continuously exposed to $>95 \%$ hyperoxia (42). Importantly, our data indicate that TrxR1 inhibition is only detrimental in settings of GSH system disruption. Work by ourselves and others suggests that a cross-talk between the Trx and GSH systems most reasonably explains the tolerance of TrxR1 inhibition in vivo and in vitro $(2,23,34,38,39,42)$. Other than dietary riboflavin deficiency that is easily correctable, GSH system deficiencies are virtually unknown in humans supporting the translational potential of ATG or other TrxR1 inhibitors for the treatment of lung injury in human patients (10-12). The fact that no obvious morbidity was seen in rheumatoid arthritis patients treated with ATG at doses for approximately 32 times greater than those used in the present studies further supports the therapeutic safety of ATG (2).

As modeled in Figure 10, we propose that exposure to LPS and hyperoxia increases free radical production by epithelia, neutrophils, and macrophages. The subsequent decrease in GSH levels, in turn, enhances pro-inflammatory cytokine and chemokine production, likely via $\mathrm{NF}-\kappa \mathrm{B}$ and/or AP-1 activation. Subsequent increases in inflammatory mediators and capillary leak lead to fatal lung injury. We propose that ATG increases pulmonary Nrf2 activation, resulting in increased production of GSH in epithelia, neutrophils, and macrophages before the accumulation of substantial lung injury. Increased GSH levels, in turn, attenuate capillary leak, decrease lung injury, and improve survival. As indicated in Figure 10, the present studies do not exclude the possibility of ATG-mediated GSH induction by Nrf2-independent mechanisms nor do they exclude a possible contribution of nonantioxidant mechanisms for protection by ATG. Future studies using this experimental model will include additional 

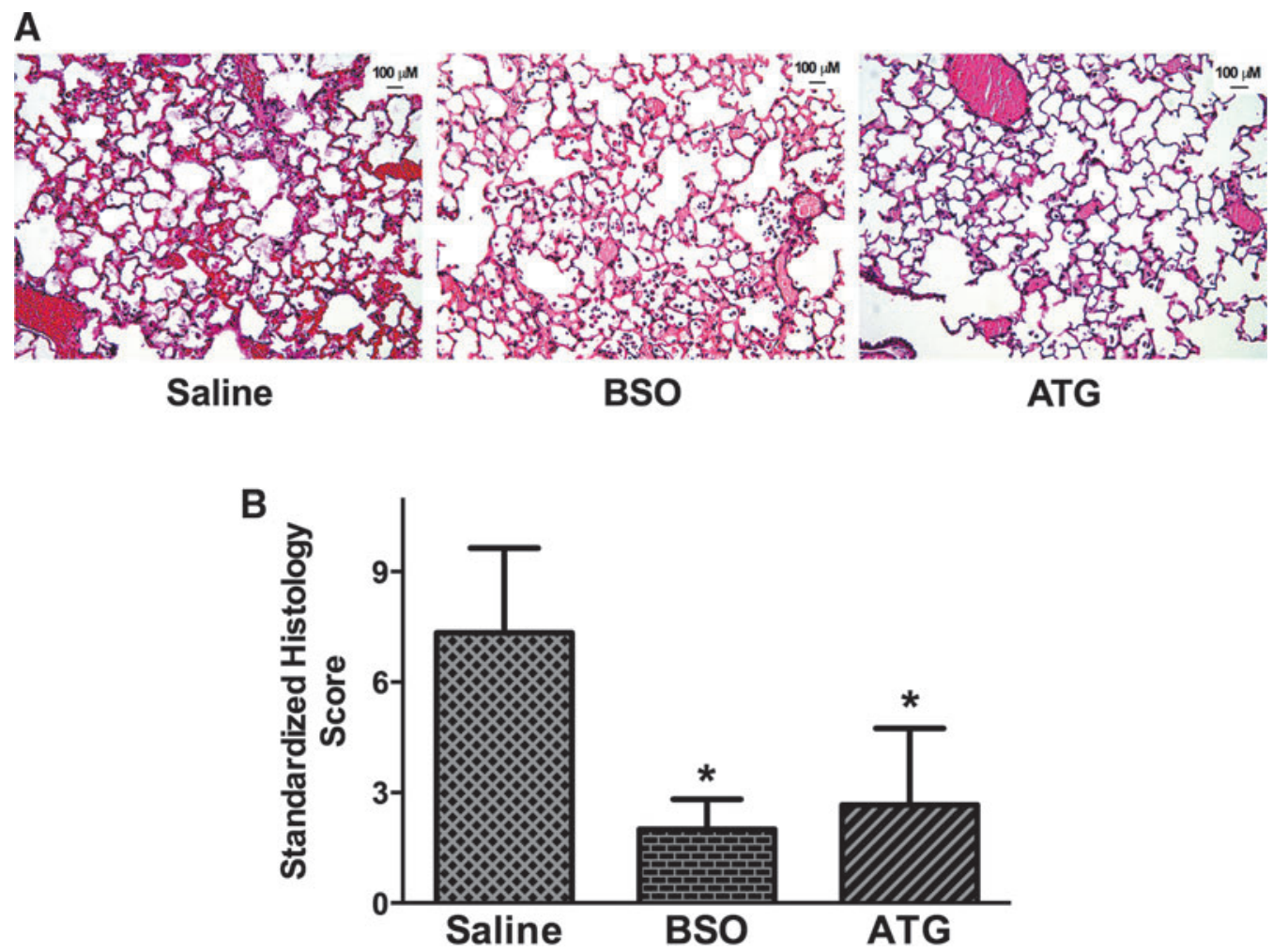

FIG. 9. Lung injury in LPS/hyperoxia-exposed mice. Adult mice were treated with a single intraperitoneal injection of saline, $25 \mathrm{mg} / \mathrm{kg} \mathrm{ATG}$, and/or $800 \mathrm{mg} / \mathrm{kg}$ BSO at $12 \mathrm{~h}$ after intratracheal LPS administration. Mice were then exposed to hyperoxia and were sacrificed at 72 h. (A) Representative lung histology (H\&E) sections. (B) Standardized lung histology score calculated as described in the Materials and Methods section. Data (mean \pm SD) were analyzed by one-way ANOVA with Tukey's multiple-comparisons test post hoc $(n=3-4)$. ${ }^{*} p=0.012$ versus saline. H\&E, hematoxylin and eosin. To see this illustration in color, the reader is referred to the web version of this article at www.liebertpub.com/ars

measurements of oxidative stress markers such as isoprostanes (30), in addition to antioxidant capacity. Noninvasive techniques based on measurement of breath markers (28), including isoprostanes $(3,26)$, leukotriene B4 (29), endogenous metabolites $(32,33)$, and volatile organic com-

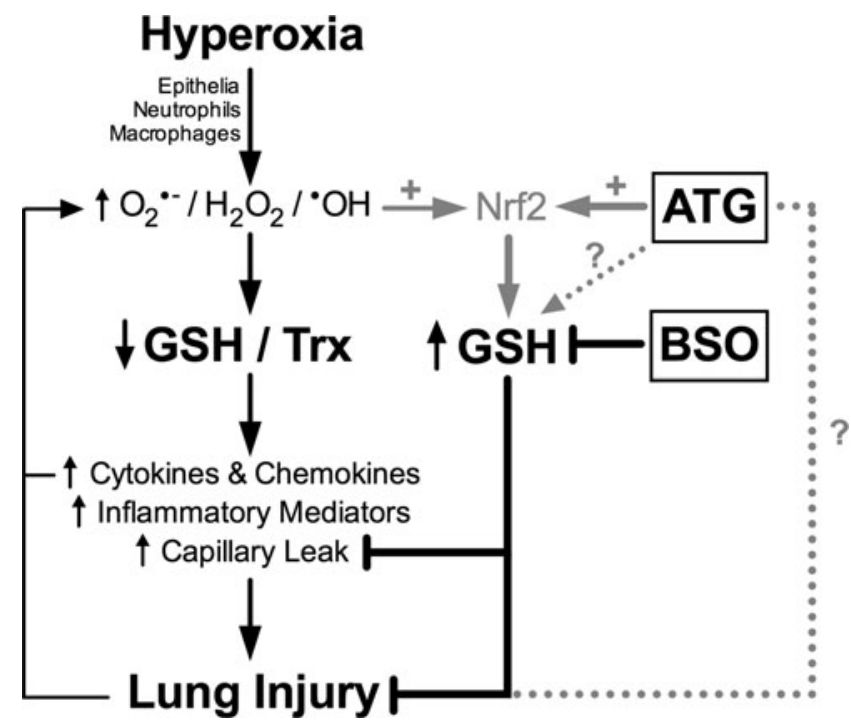

FIG. 10. Proposed scheme for the effects of ATG on inflammatory lung injury. pounds $(4,31)$, could provide a useful tool for the assessment of inflammation and/or oxidative stress in models of ARDS or in patients with ARDS.

In summary, our data demonstrate that the augmentation of GSH systems by TrxR1 inhibition with ATG protects against lung injury in a murine model of severe ARDS. If pharmacologic TrxR1 inhibition can be safely utilized to induce Nrf2 activation and increase GSH levels in critically ill patients, this novel strategy could attenuate lung injury and improve outcomes in this highly vulnerable population.

\section{Materials and Methods \\ Animal model}

Animal protocols were approved by the Institutional Animal Care and Use committee at the Research Institute at the Nationwide Children's Hospital. Mice were handled in accordance with the National Institutes of Health guidelines and housed in a pathogen-free facility. Eight- to 12-week-old adult male $\mathrm{C} 3 \mathrm{H} / \mathrm{HeN}$ mice were obtained from Harlan Laboratories (Indianapolis, IN). Mice were anesthetized with isofluorane and then, saline or $0.375 \mu \mathrm{g} / \mathrm{g}$ E. coli LPS (serotype 0111:B4, cat no. 437627; Calbiochem, Gibbstown, NJ) was instilled intratracheally (1). Twelve hours after LPS administration, mice were injected intraperitoneally (i.p.) with saline, $25 \mathrm{mg} / \mathrm{kg}$ ATG (Research Diagnostics, Flanders, $\mathrm{NJ}$ ), and/or $800 \mathrm{mg} / \mathrm{kg}$ BSO. Doses of ATG and BSO were chosen based on previous studies $(25,42)$. After medication 
administration, mice were randomly distributed into equally sized groups and either remained in RA or were exposed to hyperoxia $\left(>95 \% \mathrm{O}_{2}\right.$ ) for approximately $150 \mathrm{~h}$. In survival studies, mice were closely monitored by research personnel who were blinded to the treatment group and were euthanized when they met predetermined endpoint criteria (decreased activity, labored breathing, and inability to access food and water). Before sacrifice, mice were anesthetized with ketamine/xylazine $(200 / 20 \mathrm{mg} / \mathrm{kg}$, i.p.). Lungs were thrice lavaged with $1 \mathrm{ml}$ sterile saline to obtain BAL fluid. BAL protein concentrations were determined by Bradford assay. Cells that were recovered from BAL were counted by trypan blue exclusion. BAL supernatant, cells, and lung tissues were collected and stored at $-80^{\circ} \mathrm{C}$ until further analysis.

\section{GSH levels}

GSH levels were measured in lung tissues and in BAL cell lysates BAL by the enzyme recycling method as previously described (41).

\section{Cytokine expression}

Levels of IL-6, KC, MIP-2, TNF $\alpha$, and receptor for glycation end products (RAGE) in BAL were measured using ELISA Duoset kits (R\&D Systems, Minneapolis, MN) following the manufacturer's protocols. Absorbance was determined spectrophotometrically using a Spectramax M2 plate reader (Molecular Devices, Sunnyvale, CA).

\section{Quantitative real-time polymerase chain reaction}

RNA was isolated from homogenized lung tissues using an RNeasy mini kit (Qiagen, Valencia, CA). cDNA was synthesized using superscript reverse transcriptase III kit (Invitrogen, Carlsbad, CA) including oligo d(T) (Invitrogen) and dNTP (Fisher, Waltham, MA). Quantitative real-time polymerase chain reaction was performed using a SYBR green master mix (Qiagen), and CT values were determined using a Mastercycler EP Realplex (Eppendorf, Hauppauge, NY). $\Delta \mathrm{CT}$ values were determined by normalizing to $\beta$-actin. Primers were for murine GCLM (23), and murine $\beta$-actin (5) were obtained from Integrated DNA Technologies (San Diego, CA). Fold-change values were calculated using $2^{-(\Delta \Delta C T)}$.

\section{Tissues for histopathologic examination and scoring}

Tracheas of mice were cannulated with 25-gauge silastic catheters, and $10 \%$ buffered formalin (Fisher Scientific, Fair Lawn, $\mathrm{NJ}$ ) was instilled at $20 \mathrm{~cm} \mathrm{H}_{2} \mathrm{O}$ pressure. After $5 \mathrm{~min}$, the left lungs were removed and fixed overnight in $10 \%$ formalin. The next day, the lung samples were washed in PBS, serially dehydrated in increasing concentrations of ethanol, and then embedded in paraffin. Five-micron tissue sections were stained with H\&E. Standardized histological scoring was performed by a veterinary pathologist (Diplomate, American College of Veterinary Pathology) who was blinded to the treatment group. The three main criteria examined included interstitial change, inflammation, and consolidation. Interstitial change was broken down into perivascular change and alveolar change (each factor can score 0-4 points). Perivascular change was scored by the amount of clear space expansion surrounding large to medium caliber arteries and veins, and varied from 0 (no ex- pansion) to 4 (spaces expanded four times normal size). Alveolar change was scored by the number of alveoli with thickened septae in five randomly selected fields. Scoring ranged from 0 (no expansion) to 4 (more than $50 \%$ of walls are expanded). Inflammation was broken down into perivascular and alveolar inflammation (each factor can score 0-4 points). Perivascular inflammation was scored by the number of large and medium caliber arteries and veins that were surrounded by any mixed inflammation. Scoring ranged from 0 (no inflammation around any vessels) to 4 (all vessels have prominent inflammatory cuffs). Alveolar inflammation was scored by the number of alveoli with at least two to three inflammatory cells present in five randomly selected fields. The scores ranged from $0(0 \%)$ to $4(>50 \%)$. Consolidation is the percentage of the alveolar spaces that are collapsed in the entire lung field (ranges $0-4)$. Scores ranged from $0(0 \%)$ to 4 $(>50 \%)$. The total possible score for each lung section is 20 points (4 out of 4 in all 5 parameters).

\section{Statistics}

Data were tested for homogeneity of variances and were log-transformed when indicted. Data were analyzed using GraphPad Prism 6.0 (La Jolla, CA) by unpaired $t$-test, twoway ANOVA followed by Tukey's multiple-comparison tests, or Log-rank (Mantel-Cox) test. Significance was accepted at $p<0.05$.

\section{Acknowledgments}

The authors would like to thank Lyn Wancket, DVM, for her expert assistance with animal studies and funding from National Institutes of Health (R.D.B. F31HL097619, L.K.R. R01AT006880, and T.E.T. K08HL093365-03) and the Deutsche Forschungsgemeinschaft (M.V., VE 614/1-1).

\section{Author Disclosure Statement}

The authors declare that no competing financial interests exist.

\section{References}

1. Aggarwal NR, D'Alessio FR, Tsushima K, Files DC, Damarla M, Sidhaye VK, Fraig MM, Polotsky VY, and King LS. Moderate oxygen augments lipopolysaccharideinduced lung injury in mice. Am J Physiol Lung Cell Mol Physiol 298: L371-L381, 2010.

2. Arner ES. Focus on mammalian thioredoxin reductasesimportant selenoproteins with versatile functions. Biochim Biophys Acta 1790: 495-526, 2009.

3. Barreto M, Villa MP, Olita C, Martella S, Ciabattoni G, and Montuschi P. 8-Isoprostane in exhaled breath condensate and exercise-induced bronchoconstriction in asthmatic children and adolescents. Chest 135: 66-73, 2009.

4. Bofan M, Mores N, Baron M, Dabrowska M, Valente S, Schmid M, Trove A, Conforto S, Zini G, Cattani P, Fuso L, Mautone A, Mondino C, Pagliari G, D'Alessio T, and Montuschi P. Within-day and between-day repeatability of measurements with an electronic nose in patients with COPD. J Breath Res 7: 017103, 2013.

5. Britt RD, Jr., Locy ML, Tipple TE, Nelin LD, and Rogers LK. Lipopolysaccharide-induced cyclooxygenase-2 expression in mouse transformed Clara cells. Cell Physiol Biochem 29: 213-222, 2012. 
6. Coalson JJ, King RJ, Winter VT, Prihoda TJ, Anzueto AR, Peters JI, and Johanson WG, Jr. O2- and pneumoniainduced lung injury. I. Pathological and morphometric studies. J Appl Physiol 67: 346-356, 1989.

7. Crapo JD. Morphologic changes in pulmonary oxygen toxicity. Annu Rev Physiol 48: 721-731, 1986.

8. Crapo JD, Freeman BA, Barry BE, Turrens JF, and Young SL. Mechanisms of hyperoxic injury to the pulmonary microcirculation. Physiologist 26: 170-176, 1983.

9. Danielson DS, Heagy W, Nieman KM, and West MA. Relative hyperoxia augments lipopolysaccharide-stimulated cytokine secretion by murine macrophages. Surgery 133: 538-546, 2003.

10. el-Hazmi MA and Warsy AS. Glutathione reductase deficiency in association with sickle cell and thalassaemia genes in Saudi populations. Hum Hered 35: 326-332, 1985.

11. el-Hazmi MA and Warsy AS. Glutathione reductase in the south-western province of Saudi Arabia-genetic variation vs. acquired deficiency. Haematologia (Budap) 22: 37-42, 1989.

12. el-Hazmi MA and Warsy AS. Riboflavin status in Saudi Arabia-a comparative study in different regions. Trop Geogr Med 41: 22-25, 1989.

13. Frank L. Developmental aspects of experimental pulmonary oxygen toxicity. Free Radic Biol Med 11: 463-494, 1991.

14. Frank L, Bucher JR, and Roberts RJ. Oxygen toxicity in neonatal and adult animals of various species. J Appl Physiol 45: 699-704, 1978.

15. Frank L and Massaro D. The lung and oxygen toxicity. Arch Intern Med 139: 347-350, 1979.

16. Frank L and Massaro D. Oxygen toxicity. Am J Med 69: 117-126, 1980.

17. Freeman BA, Mason RJ, Williams MC, and Crapo JD. Antioxidant enzyme activity in alveolar type II cells after exposure of rats to hyperoxia. Exp Lung Res 10: 203-222, 1986.

18. Gromer S, Arscott LD, Williams CH Jr., Schirmer RH, and Becker K. Human placenta thioredoxin reductase. Isolation of the selenoenzyme, steady state kinetics, and inhibition by therapeutic gold compounds. J Biol Chem 273: 2009620101, 1998.

19. Haddad JJ. Glutathione depletion is associated with augmenting a proinflammatory signal: evidence for an antioxidant/pro-oxidant mechanism regulating cytokines in the alveolar epithelium. Cytokines Cell Mol Ther 6: 177-187, 2000.

20. Kim DH, Choi CW, Kim EK, Kim HS, Kim BI, Choi JH, Lee MJ, and Yang EG. Association of increased pulmonary interleukin-6 with the priming effect of intra-amniotic lipopolysaccharide on hyperoxic lung injury in a rat model of bronchopulmonary dysplasia. Neonatology 98: 23-32, 2010.

21. Knight PR, Kurek C, Davidson BA, Nader ND, Patel A, Sokolowski J, Notter RH, and Holm BA. Acid aspiration increases sensitivity to increased ambient oxygen concentrations. Am J Physiol Lung Cell Mol Physiol 278: L1240L1247, 2000.

22. Kohno M, Ishizaka A, Sawafuji M, Koh H, Hirayama Y, Ikeda E, Shiomi T, Ohashi A, Okada Y, and Kobayashi K. Hyperoxia-induced emphysematous changes in subacute phase of endotoxin-induced lung injury in rats. Am J Physiol Lung Cell Mol Physiol 287: L184-L190, 2004.
23. Locy ML, Rogers LK, Prigge JR, Schmidt EE, Arner ES, and Tipple TE. Thioredoxin reductase inhibition elicits Nrf2-mediated responses in Clara cells: implications for oxidant-induced lung injury. Antioxid Redox Signal 17: 1407-1416, 2012.

24. This reference has been deleted.

25. Lombard-Gillooly $\mathrm{K}$ and Hubbard AK. Modulation of silica-induced lung injury by reducing lung non-protein sulfhydryls with buthionine sulfoximine. Toxicol Lett 66: 305-315, 1993.

26. Lucidi V, Ciabattoni G, Bella S, Barnes PJ, and Montuschi P. Exhaled 8-isoprostane and prostaglandin E(2) in patients with stable and unstable cystic fibrosis. Free Radic Biol Med 45: 913-919, 2008.

27. Maeda T, Kimura S, Matsumoto T, Tanabe Y, Gejyo F, and Yamaguchi K. Hyperoxia accelerates Fas-mediated signaling and apoptosis in the lungs of Legionella pneumophila pneumonia. BMC Res Notes 4: 107, 2011.

28. Malerba M and Montuschi P. Non-invasive biomarkers of lung inflammation in smoking subjects. Curr Med Chem 19: 187-196, 2012.

29. Montuschi P. LC/MS/MS analysis of leukotriene B4 and other eicosanoids in exhaled breath condensate for assessing lung inflammation. J Chromatogr B Analyt Technol Biomed Life Sci 877: 1272-1280, 2009.

30. Montuschi P, Curro D, Ragazzoni E, Preziosi P, and Ciabattoni G. Anaphylaxis increases 8-iso-prostaglandin F2alpha release from guinea-pig lung in vitro. Eur $J$ Pharmacol 365: 59-64, 1999.

31. Montuschi P, Mores N, Trove A, Mondino C, and Barnes PJ. The electronic nose in respiratory medicine. Respiration 85: 72-84, 2013.

32. Montuschi P, Paris D, Melck D, Lucidi V, Ciabattoni G, Raia V, Calabrese C, Bush A, Barnes PJ, and Motta A. NMR spectroscopy metabolomic profiling of exhaled breath condensate in patients with stable and unstable cystic fibrosis. Thorax 67: 222-228, 2012.

33. Motta A, Paris D, Melck D, de Laurentiis G, Maniscalco M, Sofia M, and Montuschi P. Nuclear magnetic resonancebased metabolomics of exhaled breath condensate: methodological aspects. Eur Respir J 39: 498-500, 2012.

34. Prigge JR, Eriksson S, Iverson SV, Meade TA, Capecchi MR, Arner ES, and Schmidt EE. Hepatocyte DNA replication in growing liver requires either glutathione or a single allele of txnrd1. Free Radic Biol Med 52: 803-810, 2012.

35. Rahman I, Biswas SK, Jimenez LA, Torres M, and Forman HJ. Glutathione, stress responses, and redox signaling in lung inflammation. Antioxid Redox Signal 7: 42-59, 2005.

36. Rahman I, Yang SR, and Biswas SK. Current concepts of redox signaling in the lungs. Antioxid Redox Signal 8: 681689, 2006.

37. Rubenfeld GD, Caldwell E, Peabody E, Weaver J, Martin DP, Neff M, Stern EJ, and Hudson LD. Incidence and outcomes of acute lung injury. N Engl J Med 353: 16851693, 2005.

38. Rubinstein I, Langevitz P, and Pras M. Aurothioglucose overdosage in five patients with rheumatoid arthritis. Clin Rheumatol 6: 583-587, 1987.

39. Scarbrough PM, Mapuskar KA, Mattson DM, Gius D, Watson WH, and Spitz DR. Simultaneous inhibition of glutathione- and thioredoxin-dependent metabolism is necessary to potentiate 17AAG-induced cancer cell killing via oxidative stress. Free Radic Biol Med 52: 436-443, 2012. 
40. Su X, Looney MR, Gupta N, and Matthay MA. Receptor for advanced glycation end-products (RAGE) is an indicator of direct lung injury in models of experimental lung injury. Am J Physiol Lung Cell Mol Physiol 297: L1-L5, 2009.

41. Tipple TE and Rogers LK. Methods for the determination of plasma or tissue glutathione levels. Methods Mol Biol 889: 315-324, 2012.

42. Tipple TE, Welty SE, Rogers LK, Hansen TN, Choi YE, Kehrer JP, and Smith CV. Thioredoxin-related mechanisms in hyperoxic lung injury in mice. Am J Respir Cell Mol Biol 37: 405-413, 2007.

43. Vadasz I and Brochard L. Update in acute lung injury and mechanical ventilation 2011. Am J Respir Crit Care Med 186: 17-23, 2012.

Address correspondence to: Dr. Trent E. Tipple Center for Perinatal Research The Research Institute at Nationwide Children's Hospital 575 Children's Crossroad Columbus, $\mathrm{OH} 43215$

E-mail: trent.tipple@nationwidechildrens.org

Date of first submission to ARS Central, March 22, 2013; date of final revised submission, November 8, 2013; date of acceptance, December 2, 2013.

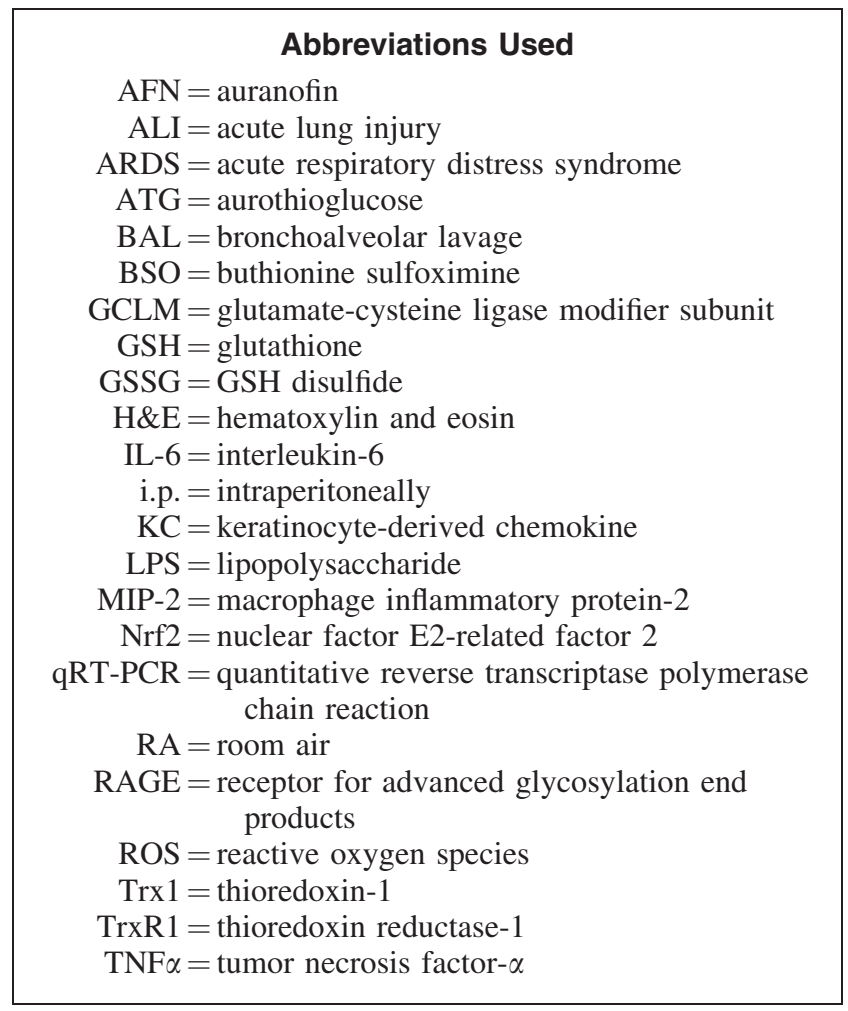

